To be published in the Proceedings of the Carolina Sym-

posium on Neutrino Physics, Columbia, SC, March 10-12, 2000.

\title{
What is coherent in neutrino oscillations The analog with a two-slit experiment
}

\author{
Harry J. Lipkin* \\ Department of Particle Physics Weizmann Institute of Science, Rehovot 76100, Israel \\ School of Physics and Astronomy, Raymond and Beverly Sackler Faculty of Exact Sciences, Tel \\ Aviv University, Tel Aviv, Israel
}

High Energy Physics Division, Argonne National Laboratory, Argonne, IL 60439-4815, USA

harry.lipkin@weizmann.ac.il

Abstract

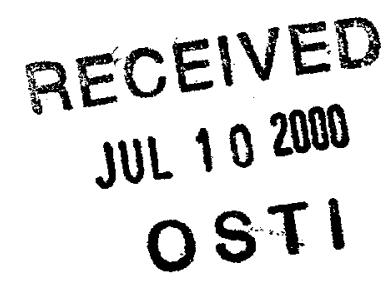

The standard treatment of neutrino oscillations recalls the story of the mathematics lecturer who said "It is obvious that..." then stopped and said; "Is it obvious?", went out for a half hour, and returned to say; "Yes it is obvious". The textbook neutrino-oscillation wave function, a coherent linear combination of states with different energies, is not found in any real experiments. Its application to reality is obvious. But the interval between "Is it obvious?" and "Yes it is obvious" is filled with many wrong arguments, many wrong papers, and more papers showing that the wrong arguments are wrong. We clarify this issue by describing the passage of a neutrino from source to detector as a multipath experiment where knowing the path destroys coherence, considering the beam and the detector as a correlated quantum system and applying this approach to Bragg scattering by $\mathrm{X}$-rays as well as neutrinos. Amplitudes with the same energy and different masses are detected coherently and produce oscillations. Amplitudes with different energies are incoherent. Quantum mechanics alone shows the existence of a neutrino mass difference to be required to explain the observed Super-Kamiokande data.

*Supported in part by grant from US-Israel Bi-National Science Foundation and by the U.S. Department of Energy, Division of High Energy Physics, Contract W-31-109-ENG-38.

The submitted manuscript has been created by the University of Chicago as Operator of Argonne National Laboratory ("Argonne") under Contract No. W-31-109-ENG-38 with the U.S. Department of Energy. The U.S. Government retains for itself, and others acting on its behalf, a paid-up, nonexclusive irrevocable worldwide license in said article 


\section{DISCLAIMER}

This report was prepared as an account of work sponsored by an agency of the United States Government. Neither the United States Government nor any agency thereof, nor any of their employees, make any warranty, express or implied, or assumes any legal liability or responsibility for the accuracy, completeness, or usefulness of any information, apparatus, product, or process disclosed, or represents that its use would not infringe privately owned rights. Reference herein to any specific commercial product, process, or service by trade name, trademark, manufacturer, or otherwise does not necessarily constitute or imply its endorsement, recommendation, or favoring by the United States Government or any agency thereof. The views and opinions of authors expressed herein do not necessarily state or reflect those of the United States Government or any agency thereof. 


\section{DISCLAIMER}

Portions of this document may be illegible in electronic image products. Images are produced from the best available original document. 


\section{INTRODUCTION - FRANK AVIGNONE AND NEUTRINO COHERENCE}

It is a pleasure to participate in this tribute to Frank Avignone. My first contact with Frank's physics intuition was when I was asked NOT TO REFEREE a paper by Frank" and Friends [1] which questioned a well-known treatment of coherence between different neutrino scattering amplitudes. The editor wanted a really impartial referee because Frank's friends were also from Israel, but he urged me to submit a companion "pedestrian" paper [2] for experimenters like him who could not understand the rigorous theorems of Frank et al. Frank insists that this paper was really the work of his friends. But Frank provided the physical intuition and understanding of what coherence was all about even though he couldn't prove himself that a paper was wrong, and provided the motivation for those who could.

The present talk investigates why and how neutrino states with different masses can be coherent. When a pion decays at rest $\pi \rightarrow \mu \nu$ the energies and momenta of the neutrino and muon are all known. This is just a "Missing Mass" experiment. The value of $M_{\nu}$ is uniquely determined by $M_{\nu}^{2}=\left(M_{\pi}-E_{\mu}\right)^{2}-p_{\mu}^{2}$. So how can there be interference between states of different mass? The answer to this paradox is found by using another paper by Frank's friends discussing which amplitudes are coherent in quantum mechanics [3].

Best wishes to Frank for continued fruitful activity for many years to come.

\section{INTERPRETING THE STANDARD TEXTBOOK WAVE FUNCTION}

The standard textbook neutrino-oscillation wave function has several states with the same momentum and different energies but has a constant magnitude over all space and is not going anywhere! The probability is the same to find a neutrino anywhere any time. But the phase difference between two waves having the same momentum and different masses $\left(m_{1}, m_{2}\right)$ and energies $\left(E_{1}, E_{2}\right)$ increases linearly with time at a rate calculated in the textbooks.

$$
\delta \phi=\left(E_{1}-E_{2}\right) t=\frac{\left(E_{1}^{2}-E_{2}^{2}\right) t}{\left(E_{1}+E_{2}\right)}=\frac{\left(m_{1}^{2}-m_{2}^{2}\right) t}{2 \bar{E}}=\frac{\left(m_{1}^{2}-m_{2}^{2}\right) x}{2 p}
$$


where we have set $\hbar=1 . \bar{E}=\left(E_{1}+E_{2}\right) / 2$ and used the velocity $v=p / \bar{E}=x / t$ to convert time into distance and obtain the OBVIOUS connection from textbook frequency to a real experiment where the $\nu$ moves from a source to a detector at a distance $x$ from the source.

IS IT REALLY OBVIOUS? The two states with the same momentum and different energies also have different velocities, $v_{1}$ and $v_{2}$ and arrive at the point $\mathrm{x}$ at different times $t_{1}$ and $t_{2}$. This suggests a different result,

$$
\delta \phi=E_{1} t_{1}-E_{2} t_{2}=\left(\frac{E_{1}}{v_{1}}-\frac{E_{2}}{v_{2}}\right) \cdot x=\frac{\left(E_{1}^{2}-E_{2}^{2}\right) x}{p}=\frac{\left(m_{1}^{2}-m_{2}^{2}\right) x}{p}
$$

The direct description of oscillations in space between states having same energy and different momenta [4] gives a result equivalent to the OBVIOUS textbook result (2.1) and disagrees with (2.2) by a factor of two.

$$
\delta \phi=\left(p_{1}-p_{2}\right) x=\frac{\left(p_{1}^{2}-p_{2}^{2}\right) x}{2 \bar{p}}=\frac{\left(m_{2}^{2}-m_{1}^{2}\right) x}{2 \bar{p}}
$$

Which is correct? What has happened between source and detector? Why same energy and different momenta? Many wrong papers have been written. Many more to show that these are wrong $[5,6]$.

\section{THE ANALOG WITH TWO-SLIT AND BRAGG SCATTERING EXPERIMENTS}

To understand which amplitudes are coherent and confirm that YES IT IS OBVIOUS and the textbook answer is $\mathrm{OK}$, consider neutrino oscillations as two-slit experiments in momentum space with a quantum detector.

In a neutrino oscillation experiment a particle passes without being observed between a source and a detector, just as in the two-slit electron diffraction experiment. In Bragg scattering a photon may be scattered by any one of the atoms in the crystal, but which atom scattered the photon is not known. In a neutrino oscillation experiment, the neutrino carrying momentum and energy from source to detector may be in any of the allowed mass 
eigenstates, but which carries this momentum and energy is not known. The amplitude at the detector is the coherent sum of the amplitudes from all allowed paths in energymomentum space. Both in Bragg scattering and neutrino oscillations there would be no coherence if the energy and momenta of all relevant particles were measured precisely and momentum conservation could determine which amplitude produced a given final state at the detector. But coherence between amplitudes is not introduced by simple ignorance of which path was taken [7]. Coherence results only from an uncertainty required by quantum mechanics.

To clarify the relation between coherence and incompleteness of knowledge consider $[8,9]$ a simple "two-slit" which-path experiment $[3,10]$ with a particle beam split into two paths and the two amplitudes, denoted by $|L(x)\rangle$ and $|R(x)\rangle$, then recombined at a point $x$ on a screen. A classical detector in one path determines the path taken and destroys all coherence. A quantum detector is a quantum system which undergoes a transition denoted by $\left|D_{i}\right\rangle \rightarrow$ $\left|D_{f}\right\rangle$, where $\left|D_{i}\right\rangle$ and $\left|D_{f}\right\rangle$ denote the initial and final states of the detector. If there is a quantum detector in the " $R$ " path, the wave function for the combined system of the particle and the detector and the intensity observed at $x$ are [5]:

$$
\begin{gathered}
\Psi(x, D)=\left|L(x), D_{i}\right\rangle+\left|R(x), D_{f}\right\rangle \\
\left.I(x)=|| L(x)\rangle\left.\right|^{2}+|| R(x)\right\rangle\left.\right|^{2}+2 \operatorname{Re}\left[|\langle L(x) \mid R(x)\rangle| \cdot e^{i \theta(x)} \cdot\left\langle D_{i} \mid D_{f}\right\rangle\right]
\end{gathered}
$$

where $\theta(x)$ is relative phase of $|L(x)\rangle$ and $|R(x)\rangle$ and its variation with $x$ gives the interference pattern on the screen.

The quantum detector introduces an additional factor and phase into the interference term, given by the detector overlap $\left\langle D_{i} \mid D_{f}\right\rangle$.

Bragg scattering is a which-path experiment with many paths, one for each scattering atom, and a quantum detector in each path. The detector is the full lattice and each interference term between two paths contains two coherence factors $\left\langle D_{i} \mid D_{f}\right\rangle$ that depend on the lattice dynamics. The probability $P_{D W}$ that the scattering is coherent is called the 
"Debye-Waller" factor $[10,11], P_{D W}=\left|\left\langle D_{i} \mid D_{f}\right\rangle\right|^{2}$ which defines the relative amounts of coherent Bragg scattering and incoherently scattered photons observed outside the Bragg peak.

\section{DETAILED QUANTUM MECHANICS OF A $\nu$ SOURCE AND DETECTOR}

We now apply the general which-path formalism developed above to a neutrino - detector system, where a neutrino $\nu_{k}$ with energy, mass and momentum $E_{\nu}, m_{k}$ and $\vec{P}_{k}$ is detected via the transition $\nu_{k}+n \rightarrow \mu^{-}+p$ occurring on a neutron in the detector, The detector transition matrix element is

$$
\left\langle D_{k f}|T| D_{i}\right\rangle=\left\langle D_{k f}\left|I_{+} e^{i\left(\vec{P}_{k}-\vec{P}_{\mu}\right) \cdot \vec{X}}\right| D_{i}\right\rangle
$$

where $D_{i}$ and $D_{k f}$ denote the initial detector state and the final state produced in the "path $k$ "; i.e. after the absorption of a neutrino with mass $m_{k}$ and emission of a $\mu^{-}$with energy $E_{\mu}$ and mmentum $\vec{P}_{\mu}, \vec{X}$ denotes the neutron co-ordinate and $I_{+}$denotes the charge exchange isospin operator.

The overlap between the final detector wave functions after the transitions absorbing neutrinos with masses $m_{k}$ and $m_{j}$ is then

$$
\left\langle D_{k f} \mid D_{j f}\right\rangle=\left\langle D_{i}\left|e^{i\left(\vec{P}_{j}-\vec{P}_{k}\right) \cdot \vec{X}}\right| D_{i}\right\rangle
$$

If the quantum fluctuations in the position of the active nucleon in the initial state of the detector are small in comparison with the oscillation wave length, $\hbar /\left(\vec{P}_{j}-\vec{P}_{k}\right)$, there is effectively a full overlap between final detector states after absorption of different mass neutrinos, and a full coherence between neutrino states with the same energy and different momenta.

$$
\begin{gathered}
\left|\vec{P}_{j}-\vec{P}_{k}\right|^{2} \cdot\left\langle D_{i}|| \vec{X}^{2}|| D_{i}\right\rangle \ll 1 \\
\left\langle D_{k f} \mid D_{j f}\right\rangle \approx 1-(1 / 2) \cdot\left|\vec{P}_{j}-\vec{P}_{k}\right|^{2} \cdot\left\langle D_{i}|| \vec{X}^{2}|| D_{i}\right\rangle \approx 1
\end{gathered}
$$


The total energies of the final muon and detector produced after absorption of neutrinos with different energies are different. These muon-detector states are thus orthogonal to one another and there is no coherence between detector states produced by the absorption of neutrinos with different energies.

\section{SIMPLE QUANTUM MECHANICS AND SUPER-KAMIOKANDE}

Simple quantum mechanics alone, without the full apparatus of the standard model, shows that the Super-Kamiokande results [12] require the existence of two different mass eigenstates for neutrinos. The energy spectrum of atmospheric neutrinos cannot change between their source at the top of the atmosphere and their detection in a detector on earth if neutrinos are not absorbed, do not decay and interactions conserve energy. If there is only one neutrino mass value, the energy and momentum spectra will be identical for the upward and downward going neutrinos incident on the detector and no difference can be observed. The observation of the up-down difference [12] therefore indicates that there are at least two different mass eigenstates, and that the difference can arise from interference between the waves of states having different masses and therefore different momenta if they have the same energy.

This conclusion depends only upon quantum mechanics and previous experimental observations that $\nu_{e}$ and $\nu_{\mu}$ are orthogonal. It does not depend on the details of the standard model and remains valid even if there is new physics beyond the standard model that does not violate quantum mechanics.

\section{ACKNOWLEDGMENTS}

It is a pleasure to thank Eyal Buks, Maury Goodman, Yuval Grossman, Moty Heiblum, Yosef Imry, Boris Kayser, Lev Okun, David Sprinzak, Ady Stern and Leo Stodolsky for helpful discussions and comments. 


\section{REFERENCES}

[1] Y. Aharonov, F. T. Avignone, III, A. Casher and S. Nussinov, Phys. Rev. Lett. 58 (1987) 1173

[2] Harry J. Lipkin, Phys. Rev. Lett. 58 (1987) 1176

[3] Ady Stern, Yakir Aharonov and Yoseph Imry, Phys Rev. A41 (1990) 3436

[4] H.J. Lipkin, Phys. Lett. B 348 (1995) 604.

[5] Harry J. Lipkin, hep-ph/9907551, Physics Letters B 477 (2000) 195 and references therein

[6] Harry J. Lipkin, hep-ph/9901399, in Proceedings of the Europhysics Neutrino Oscillation Workshop (NOW'98) 7-9 September 1998. Amsterdam. Published in http://www.nikhef.nl/pub/conferences/now98/

[7] Kurt Gottfried, Am. J. Phys. 68 (2000) 143

[8] E. Buks et al, Nature 391 (1998) 871

[9] D. Sprinzak, E. Buks, M. Heiblum and H. Shtrikman, cond-mat/9907162

[10] H.J. Lipkin, Phys.Rev. A42 (1990) 49

[11] H.J. Lipkin, Hyperfine Interactions, 72 (1992) 3.

[12] Y. Fukuda et al Super-Kamiokande Coll. Phys. Rev. Lett. 81 (1998) 1562 